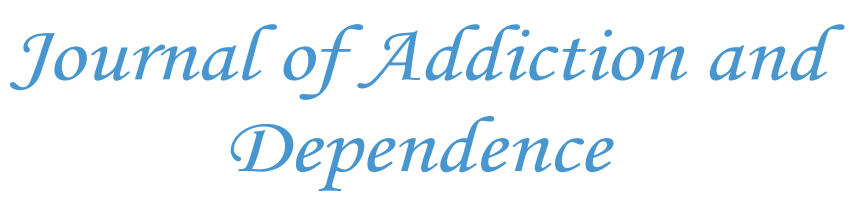

\title{
The School-Level Factors Associated with Internet Addiction among Adolescents: Across-Sectional Study in Bangladesh
}

\author{
Dilshad Afrin, Mahabub-Ul Islam, Fazle Rabbiand, Ahmed Hossain*
}

Department of Public Health, North South University, Canada

*Corresponding author: Ahmed Hossain, Department of Public Health, North South University, Canada,

E-mail: ahmed.hossain@utoronto.ca

\begin{abstract}
Objectives: The adolescents have increased the use of the Internet not ably over the last few years. Here we investigate the prevalence of Internet addiction among adolescents and identify the school-level factors on Internet addiction.

Materials and Methods: This was a school-based cross-sectional study with 279 students aged 14 - 17years in the education year of 2016. The students Internet addiction was determined through the Ormans test, which consists of nine questions.

Results: The prevalence of severe internet addiction among the surveyed adolescents was $2.51 \%$, and moderate internet addiction was $64.87 \%$. The study revealed from the multivariatelogisticmodel that the male students are $31 \%(\mathrm{OR}=0.69$, confidence interval: 0.36 - 1.34) less likely to be moderate/severely internet addicted compared to female students. Similarly, considering the number of siblings, lonely child in a house is 3.12 times $(\mathrm{OR}=3.12, \mathrm{CI}: 1.14-9.29)$ more likely to have moderate/severe internet addiction compared to who has 3ormore siblings. The students with English medium studies $(\mathrm{OR}=0.58)$ and the students who play out door games $(\mathrm{OR}=0.56)$ are also negatively associated with severe internet addiction.

Conclusion: The results indicate that the adolescents of grade nine students, Bangla medium studies and the students who don't play outside games are more prone to be the Internet addicted. Besides, female students are more in risk to be Internet addicted than male students. The findings will help us for strategies to be put in the school to reduce the Internet addiction among adolescents.
\end{abstract}

Received Date: September 13, 2017

Accepted Date: November 30, 2017

Published Date: December 06, 2017

Citation: Hossain, A.et al. The SchoolLevel Factors Associated with Internet Addiction among Adolescents: Across-Sectional Study in Bangladesh. (2017) J Addict Depend 3(2): 170- 174.

\section{DOI: $10.15436 / 2471-061 X .17 .1686$}

Keywords: Adolescents; Multivariate logistic regression; Internet addiction; Bangladesh

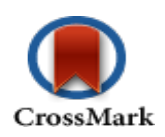

\section{Introduction}

Nowadays the most readily available media to adolescents is the internet which in the name of academic and other purposes has compelled them to use it more. This media has not only become a good source of information regarding knowledge but also for other purposes like social communication, gaming, entertainment and so on. Human nature being more tilted to this enjoy menthes enhanced the use of the Internet. Adolescents appear to be the population at risk in having internet addiction due to the variability in developing their cognitive control and boundary setting skills ${ }^{[1,2]}$. Loneliness, staying up late, tiredness, and missing morning classes were also inter-correlated with Internet-caused impairment. In a study, Nayanika and Barmo- laexamine the inter relationships among internet addiction, mental health, and academic performances among 100 high school students ${ }^{[3]}$. They found that the students who had severe internet addiction were in a poor academic performance and mental health problems. The use of the Internet on school campuses and in society has increased dramatically in recent years ${ }^{[4]}$. Adolescents are more interested in the newest technology; they use the internet more often than other age groups. Excessive use of internet among adolescents, who are not yet psychologically matured enough and trying to adapt the social environment, has put them at risk for internet addiction ${ }^{[5]}$. Heavier Internet use for entertainment was shown to be correlated highly with impaired academic performance. Research among European adolescents 
revealed that: $1.2 \%$ of the total sample presents with Internet addiction behavior, while $12.7 \%$ are at risk of developing internet addiction behavior ${ }^{[6]}$. According to the data by Internet World Stats (2016), Asia has the highest number of internet users in the world and it is $44 \%$ of the total internet users all over the world. It has been considered as a severe public health issue in certain Asian countries. In Beijing, it is found that $90 \%$ of adolescent crime was internet-related ${ }^{[7]}$. Law studied the level of Internet addiction with different age groups of 413 undergraduates and found the level of Internet addiction is in dependent of gender and major of study ${ }^{[8]}$. In fact, younger Internet users are more at the risk of becoming internet addicts than $=$ users of older age ${ }^{[9]}$. The number of internet users in Bangladesh has now been crossed 60 million, according to the 2016 statistics of telecoms regulator of which $35 \%$ are adolescents. The average size of a secondary school class in Bangladesh is approximately 50 students indicates that there may be a number of adolescents in every classroom who are struggling with moderate to severe Internet addiction. To promote the best possible outcomes for students, there is a great need for early identification of the factors that are associated with the Internet addiction. There were no studies been conducted with Bangladeshi students to identify the prevalence of Internet addiction among adolescents and the factors associated with the Internet addiction? This study aims to identify the current prevalence rate of Internet addiction and to determine the related school-level factors among secondary students of schools where easy and quick access to the internet is provide.

\section{Data and Methods}

\section{Study Area}

The study was conducted in Ispahani Public School and College (IPSC) and Chittagong Grammar School (CGS), located in Chittagong. Chittagong is the port city and financial center in south eastern Bangladesh. Both the schools are well known private educational institutions in Chittagong and offer primary, secondary and higher secondary education.

\section{Data}

The current study is based on all the secondary students. There are about 350 secondary students enrolled both in IPSC and CGS according to the registration on $20^{\text {th }}$ May2016. We conducted a population-based cross-sectional survey of the secondary students and collected data from 279 students. Other 71 students either declined to give the interview or they were absent in the school during the survey.

\section{Internet Addiction Test}

Orman's Internet Addiction Survey ${ }^{[10]}$ is a test made up of 9 questions with "Yes" or "No" answers. Following the Ormans Internet Addiction Survey, the level of Internet addiction was determined by adding the total number of "Yes" answers in these to nine questions that make up the test. A number above 7 indicates severe internet addiction; 3points or below would show an absolute absence of addiction; while an intermediate score from 4 - 6 points indicate that the student has a moderate internet addiction. Recently, Malts also recommended the use of Orman test in a study to define the Internet addiction ${ }^{[11]}$.

\section{Questionnaire survey}

An interview was designed by a semi-structured questionnaire to obtain information on Age, sex (male or female), education field (science or others), number of siblings, family structure (nuclear or joint), monthly family income ( $\geq 50000$ or $<50000$ BDT), type of school (Bangla or English medium), playing games outside (yes or no), sleep duration, physical exercise (yes or no), type of phone use (smart or simple), type of computer use(laptop, desktop or others). Sleep duration was categorized as $<6$ hours and $6-8$ hours or $8-10$ hours. In September 2016, all data was collected. Any question or confusion from the students was clarified to ensure that every student understood all the questions during the survey.

\section{Ethical approval}

Ethical approval for the study protocol was obtained from the North South University Review Committee, IPSC and CGS. A written informed consent was also obtained from all the participants.

\section{Statistical Analysis}

We analyzed the data using R. Descriptive statistics were used to evaluate all the variables. The unadjusted dissociation between each of the categorical variables and internet addiction was evaluated by Chi-square test. We designed a multivariate logistic regression model, which was adjusted for age, sex, education field, the number of siblings, family structure, monthly family income type of school, playing games outside, sleep duration, physical exercise, type of phone use, type of computer use in the model. The results were interpreted by odds ratios (ORs), 95\% Confidence Intervals (CIs) and corresponding p-values. The variables of $p$-values less than 0.05 were considered as statistically significant.

\section{Results}

\section{Descriptive statistics}

The data comprised of 279 secondary school students aged 14 - 17 years of which 118 were male and 161 were female students. The mean age of adolescent's was 15.63 years. Figure 1 presents the percentage of students with positive responses for the Orman test questions. The 190 students of the responded $(68.1 \%)$ found it is hard for them to stay away from the internet for several days at a time (question-4). Most of the students answered positively to questions 1,4 and 9. Figure 2 presents the prevalence rate of no, moderate and severe internet addiction according to the Orman's Internet addiction scale. Among the surveyed adolescents we found $2.51 \%$ are severely Internet addicted, and $64.87 \%$ have the moderate Internet addiction. We made two groups of the students for comparing between no Internet addiction and moderate/severe Internet addiction. The baseline characteristics of the participants are described in the Table 1, for comparing between no and moderate/severe Internet addiction. It appears that $61.8 \%$ of the male and $71.4 \%$ female students are moderate to severe Internet users. Besides, $74.8 \%$ of the grade nine students and $39.6 \%$ of the grade ten students are moderate to severe Internet users. The results of the unadjusted analysis by the $\chi 2$ test indicate that the grades, medium of studies, playing outside, and type of phone and computer use are significant variables to develop the moderate/severe internet addiction among the adolescents. 


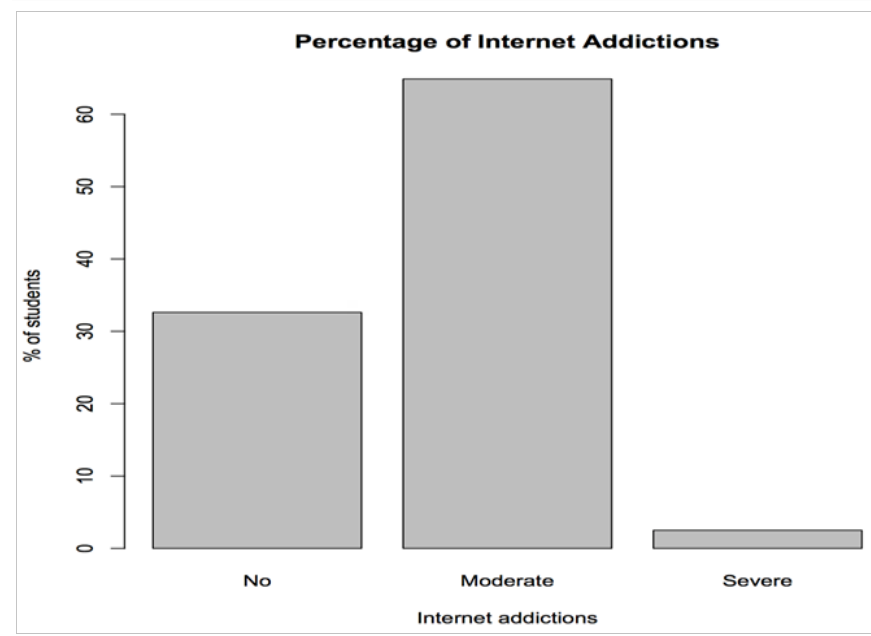

Figure 1: Barplotshows percentage of secondary students with levels of Internet addictions.

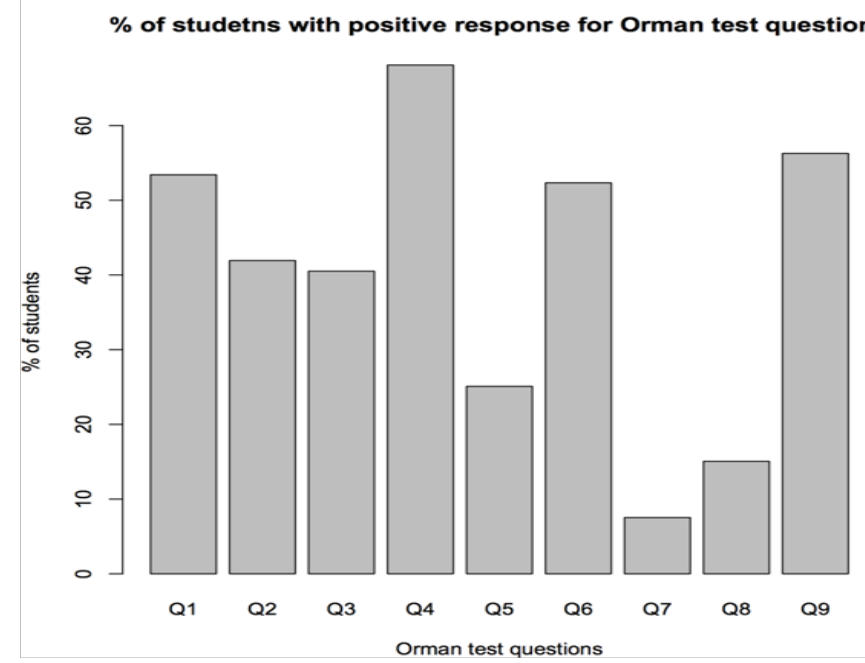

Figure 2: Percentage of students with positive response for Orman test questions. It appears that 150 students spend more time insuring the internet (Q1); 125 students feel that they have a problem in limiting the time spent on the internet (Q2); 120 students told their friends or family members complained about their time spending with computer (Q3); More than 150 students find it hard to stay away from the internet for several day set a time (Q4); 70 students feel either their workout put or personal relationships suffered as a result of spending too much time on the internet (Q5); 140 students said particular areas on the internet, or types of files, sites, they find hard to avoid (Q6); a few number of students, 30 in number said that they had trouble to control their impulses to purchase items, products, or services on the internet (Q7); 40 students tried, but were unsuccessful to curtail their use of the internet (Q8); 160 students derive much of their pleasure and satisfaction in life from being on the Internet (Q9).

\section{Multivariate Logistic Regression Model}

We fitted a multivariable logistic regression model for the Internet addiction after adjust in gall the potential factors. The adjusted odds ratios (ORs) are given in Table 2. It appears that grade ten students are $60 \%$ less likely to be the Internet addicted compared to grade nine $(\mathrm{OR}=0.4)$. This may be because class ten students are having more academic load than class nine students .Male students are $31 \%$ less likely to be the Internet addicted compared to female students though it is not significant at 5\% significance level. Similarly, the single child is 3.12 times more likely to be Internet addicted than the students who have $\geq$ 3 siblings $(\mathrm{OR}=3.12$, pvalue $=0.031)$. The medium of studies and playing outside are also two important associated factors because the set woo variables are very close to the significant level of 0.05 . This shows that the students who play outside regularly are $44 \%$ less likely to be the Internet addicted compared to the students who don't play outside $(\mathrm{OR}=0.56)$.

Table 1; Characteristics of secondary students and unadjusted analysis internet addiction.

\begin{tabular}{|c|c|c|c|c|c|}
\hline Variables & Categories & $\begin{array}{l}\text { No } \\
\text { (91) }\end{array}$ & $\begin{array}{l}\text { Yes } \\
\text { (188) }\end{array}$ & $\chi^{2}$ & $\begin{array}{l}\text { p- } \\
\text { value }\end{array}$ \\
\hline \multirow{2}{*}{ Sex } & Male & 45 & 73 & 2.41 & 0.12 \\
\hline & Female & 46 & 115 & & \\
\hline \multirow{2}{*}{ Grade } & Nine & 34 & 101 & 5.93 & 0.01 \\
\hline & Ten & 57 & 87 & & \\
\hline \multirow{2}{*}{$\begin{array}{l}\text { Medium of } \\
\text { studies }\end{array}$} & Bangla & 37 & 108 & 6.26 & 0.01 \\
\hline & English & 54 & 80 & & \\
\hline \multirow{2}{*}{ Education field } & Science & 74 & 138 & 1.69 & 0.19 \\
\hline & Others & 17 & 50 & & \\
\hline \multirow{3}{*}{ No. of Siblings } & 1 & 8 & 26 & 2.31 & 0.31 \\
\hline & 2 & 52 & 111 & & \\
\hline & $\geq 3$ & 31 & 51 & & \\
\hline \multirow{2}{*}{ Family Type } & Joint & 8 & 22 & 0.28 & 0.59 \\
\hline & Nuclear & 83 & 166 & & \\
\hline \multirow{2}{*}{ Family income } & $<50000$ & 34 & 85 & 1.24 & 0.26 \\
\hline & $>50000$ & 57 & 103 & & \\
\hline \multirow{2}{*}{ Play out side } & No & 41 & 114 & 5.41 & 0.01 \\
\hline & Yes & 50 & 74 & & \\
\hline \multirow{3}{*}{ Sleeping hour } & $4-6$ & 14 & 40 & 2.46 & 0.29 \\
\hline & $6-8$ & 62 & 110 & & \\
\hline & above 8 & 15 & 38 & & \\
\hline \multirow{2}{*}{ Exercise } & No & 47 & 44 & 0.08 & 0.76 \\
\hline & Yes & 92 & 96 & & \\
\hline \multirow{2}{*}{ Internet at home } & No & 15 & 76 & 0.33 & 0.56 \\
\hline & Yes & 38 & 150 & & \\
\hline \multirow{3}{*}{ Phone type } & None & 3 & 9 & 7.01 & 0.03 \\
\hline & Simple & 17 & 62 & & \\
\hline & Smart phone & 71 & 117 & & \\
\hline \multirow{3}{*}{ Computer type } & Desktop & 37 & 69 & 5.15 & 0.07 \\
\hline & Laptop & 33 & 92 & & \\
\hline & Others (Tabetc.) & 21 & 27 & & \\
\hline
\end{tabular}


Factors Associated with Internet Addiction

Table 2: Adjusted relationship between covariates and internet addiction that is analyzed using multivariate logistic regression.

\begin{tabular}{|l|l|l|l|l|l|}
\hline Variables & References & Estimate & OR & Confidence Interval & p-value \\
\hline Age & & -0.02 & 0.97 & $(0.63-1.48)$ & 0.9 \\
\hline Sex-Male & Female & -0.35 & 0.69 & $(0.36-1.34)$ & 0.27 \\
\hline Grade-Ten & Nine & -0.91 & 0.4 & $(0.21-0.71)$ & 0.002 \\
\hline Medium of studies- English & Bangla & -0.53 & 0.58 & $(0.31-1.06)$ & 0.079 \\
\hline Education field- Science & others & -0.12 & 0.88 & $(0.43-1.75)$ & 0.731 \\
\hline Sibling- 1 & $\geq 3$ & 1.14 & 3.12 & $(1.14-9.29)$ & 0.031 \\
\hline 2 & $\geq 3$ & 0.58 & 1.8 & $(0.96-3.40)$ & 0.066 \\
\hline Family Type Nuclear & Joint & -0.07 & 0.92 & $(0.34-2.32)$ & 0.878 \\
\hline Family income- $\geq 50000$ & $<50000$ & -0.21 & 0.8 & $(0.44-1.45)$ & 0.472 \\
\hline Play out side Yes & No & -0.56 & 0.56 & $(0.29-1.07)$ & 0.084 \\
\hline Sleeping hour- $6-8$ & $<6$ & -0.36 & 0.69 & $(0.30-1.53)$ & 0.375 \\
\hline $8-10$ & $<6$ & 0.04 & 1.04 & $(0.80-2.85)$ & 0.931 \\
\hline Exercise Yes & No & 0.36 & 1.44 & $(0.45-2.11)$ & 0.224 \\
\hline Internet at home Yes & No & -0.01 & 0.99 & $(0.28-9.40)$ & 0.98 \\
\hline Phone type-Simple & None & 0.56 & 1.75 & $(0.12-3.71)$ & 0.519 \\
\hline Smart phone & None & -0.28 & 0.75 & $(0.71-2.56)$ & 0.359 \\
\hline Computer type-Laptop & Desktop & 0.29 & 0.52 & $1.17)$ & 0.119 \\
\hline Others (Tab etc.) & Desktop & -0.65 & & \\
\hline
\end{tabular}

\section{Discussions}

Adolescents with Internet addiction have negative influences on their daily routines, academic performance and relationship with family members and friends. Therefore, preventive measure against Internet addiction should be taken by considering few school factors to guarantee better quality and more equitable student performance. In our study we considered the students of grades $9-10$. We chose to conduct the study in a younger population as adolescents of this group are getting access to Internet use frequently at school and at home.

The prevalence of Internet addiction for female students is found higher than male students. It may be because off male students have fewer opportunities of involvement in co-curricular activities in the school. But the results is opposite of the results from Pravakaran et al. study ${ }^{[1]}$. Adolescent's who don't have any siblings are found more at risk of developing an Internet addiction. Therefore family should give more time to this group of adolescents to reduce the duration of Internet use. Grade nine students are found more Internets addict compared to grade ten students. It may be because the grade nine students have less academic involvements than grade ten students. Besides the schools should give importance to the students of Bangla medium studies by creating an effective atmosphere conductive to learning without the use of the Internet.

This study was a cross-sectional study, which limits the interpretation to factors associated with Internet addiction. Besides, associated factors contributing to Internet addiction, longitudinal studies need to be planned. There could be information bias in the current study as the information was collected using self-reported questionnaires. A convenient sampling was used to choose the two schools, which limits the generalizability of these results.

\section{Conclusions}

The paper is the first of its kind of research especially with Bangladeshi adolescents and significantly increases our understanding of the school-level factors associated with Internet addiction. Guidelines for interventions need to be developed to address the Internet addiction among adolescents at the primary health-care level. Characteristics of Internet usage found to be associated with Internet addiction needs to be considered while developing strategies for interventions. Monitoring of hours and purpose of Internet usage by parents and school authorities may help in controlled Internet use.

Competing Interests: The authors declare that there is no conflict of interest regarding the publication of this paper.

Author's contributions: AH participated in study conception, design, performed statistical analysis, data interpretation and drafted the manuscript. DA contributed in study conception, design, and data collection and helped to draft the manuscript. FR contributed in draft the manuscript. MI contributed in data collection and reviewed the manuscript. All authors approved of the final manuscript.

Acknowledgements: AH, DA, MI and FR acknowledge North South University, IPSC, CGS and participants for providing us the information to conduct the study. We would also like to thank two anonymous reviewers and the editor for insightful comments that improved the presentation and clarity of our manuscript. 


\section{References}

1. Prabhakaran, M.C., Patel, V.R., Ganjiwale, D.J., et al. Factors associated with internet addiction among school-going adolescents in Vadodara. (2016) J Family Med Prim Care 5(4): 765-769.

Pubmed | Crossref | Others

2. Kuss, J.D., vanRooij, J.A., Shorter, W.G., et al. Internet addiction in adolescents: Prevalence and risk factors. (2013) Computers in Human Behavior 29(5): 1987-1996.

Pubmed | Crossref| Others

3. Singh, N., Barmola, K.C. Internet Addiction, Mental Health and Academic Performance of School Students/Adolescents. (2015) The International Journal of Indian Psychology 2(3): 100.

Pubmed|Crossref| Others

4. Marahatta, S.B., Adhikari, B., Aryal, N., et al. Internet Addiction and Associated Factors among Health Sciences Students in Nepal. (2015) J Community Med Health Educ 5:362.

Pubmed $\mid$ Crossref $\mid$ Others

5. Evren, C., Dalbudak, E., Evren, B., et al. High risk of internet addiction and its relationship with life time substance use, psychological and behavioral problems among 10th grade adolescents. (2014) Psychiatr Danub 26(4): 330-339.

Pubmed | Crossref | Others
6. Flora, K. Internet addiction disorder among adolescents and young adults: the picture in Europe and prevention strategies. (2012) Perspectives on youth Chapter10:119.

Pubmed | Crossref| Others

7. Alam, S.S., Nik, M., Hashim, N.H., et al. Negative and positive impact of internet addiction on young adults: Empirical study in Malaysia. (2014) Intangible Capital 10(3): 619-638.

Pubmed|Crossref|Others

8. Sally, L.P.M. Prediction of Internet Addiction for Undergraduates in Hong Kong. (2006).

Pubmed | Crossref $\mid$ Others

9. Sharma, A., Sahu, R., Sharma, R., et al. Internet addiction among professional courses students. (2014) Int J Med Sci Public Health 3(9):1069-1073.

Pubmed | Crossref | Others

10. Orman, M.C. What To Do If You Are (Or Fear That You May Become) Addicted To The 'Net. (1996) Internet Stress Survey. Pubmed | Crossref| Others

11. Matas, A. Ormans Internet Addiction Survey: A preliminary psychometric study in an university Andalusian sample. (2015) Univ Psychol 14(3): 1107-1116.

Pubmed | Crossref $\mid$ Others
Ommega Online Publishers

Journal Title: Journal of Addiction and Dependence(JAD)

Journal Short Name: J Addict Depend
ISSN no: 2471-061X

E-mail: addiction.depend@ommegaonline.org

Website: www.ommegaonline.org 